\title{
REQUIRING THAT MINIMAL SEPARATORS INDUCE COMPLETE MULTIPARTITE SUBGRAPHS
}

\author{
Terry A. McKeE \\ Department of Mathematics and Statistics \\ Wright State University \\ Dayton, Ohio 45435 USA \\ e-mail: terry.mckee@wright.edu
}

\begin{abstract}
Complete multipartite graphs range from complete graphs (with every partite set a singleton) to edgeless graphs (with a unique partite set). Requiring minimal separators to all induce one or the other of these extremes characterizes, respectively, the classical chordal graphs and the emergent unichord-free graphs. New theorems characterize several subclasses of the graphs whose minimal separators induce complete multipartite subgraphs, in particular the graphs that are 2-clique sums of complete, cycle, wheel, and octahedron graphs.
\end{abstract}

Keywords: minimal separator, complete multipartite graph, chordal graph, unichord-free graph.

2010 Mathematics Subject Classification: 05C62, 05C75, 05 C69.

\section{REFERENCES}

[1] A. Brandstädt, V.B. Le and J.P. Spinrad, Graph Classes: A Survey (Society for Industrial and Applied Mathematics, Philadelphia, 1999). doi: $10.1137 / 1.9780898719796$

[2] R.C.S. Machado, C.M.H. de Figueiredo and N. Trotignon, Complexity of colouring problems restricted to unichord-free and \{square, unichord $\}$-free graphs, Discrete Appl. Math. 164 (2014) 191-199.

doi:10.1016/j.dam.2012.02.016

[3] R.C.S. Machado, C.M.H. de Figueiredo and K. Vušković, Chromatic index of graphs with no cycle with a unique chord, Theoret. Comput. Sci. 411 (2010) 1221-1234. doi:10.1016/j.tcs.2009.12.018

[4] T.A. McKee, Independent separator graphs, Util. Math. 73 (2007) 217-224. 
[5] T.A. McKee, Minimal vertex separators and 3-skein subgraphs, Bull. Inst. Combin. Appl. 72 (2014) 19-24.

[6] T.A. McKee, A new characterization of unichord-free graphs, Discuss. Math. Graph Theory 35 (2015) 765-771.

doi:10.7151/dmgt.1831

[7] T.A. McKee and F.R. McMorris, Topics in Intersection Graph Theory (Society for Industrial and Applied Mathematics, Philadelphia, 1999). doi:10.1137/1.9780898719802

[8] N. Trotignon and K. Vušković, A structure theorem for graphs with no cycle with a unique chord and its consequences, J. Graph Theory 63 (2010) 31-67. doi:10.1002/jgt.20405

Received 28 June 2016

Revised 9 November 2016

Accepted 9 November 2016 